\title{
Optimal Network Topologies for Local Search with Congestion
}

\author{
R. Guimerà, ${ }^{1}$ A. Díaz-Guilera, ${ }^{2,1}$ F. Vega-Redondo, ${ }^{3,4}$ A. Cabrales, ${ }^{4}$ and A. Arenas ${ }^{5}$ \\ ${ }^{1}$ Departament d'Enginyeria Química, Universitat Rovira i Virgili, 43007 Tarragona, Spain \\ ${ }^{2}$ Departament de Física Fonamental, Universitat de Barcelona, 08028 Barcelona, Spain \\ ${ }^{3}$ Departament de Fonaments d'Anàlisi Econòmica, Universitat d'Alacant, 03071 Alacant, Spain \\ ${ }^{4}$ Departament d'Economia i Empresa, Universitat Pompeu Fabra, 08005 Barcelona, Spain \\ ${ }^{5}$ Departament d'Enginyeria Informàtica i Matemàtiques, Universitat Rovira i Virgili, 43007 Tarragona, Spain
}

(Received 9 July 2002; published 21 November 2002)

\begin{abstract}
The problem of searchability in decentralized complex networks is of great importance in computer science, economy, and sociology. We present a formalism that is able to cope simultaneously with the problem of search and the congestion effects that arise when parallel searches are performed, and we obtain expressions for the average search cost both in the presence and the absence of congestion. This formalism is used to obtain optimal network structures for a system using a local search algorithm. It is found that only two classes of networks can be optimal: starlike configurations, when the number of parallel searches is small, and homogeneous-isotropic configurations, when it is large.
\end{abstract}

DOI: $10.1103 /$ PhysRevLett.89.248701

PACS numbers: 89.75.Hc, 02.60.Pn, 02.70.Ns, 05.70.Jk

Recently, the study of topological and dynamical properties of complex networks has received a lot of interest [1-3]. Part of this interest comes from the attempt to understand the topology and behavior of computer based communication networks such as the Internet [4] and the World Wide Web [5,6]. However, the study of communication processes in a wider sense is of interest in other fields, remarkably the design of organizations [7-9].

One of the general principles that has been discovered in many such complex networks is the short average distance between nodes [1]. More surprisingly, it has been shown that these short paths can be found with essentially local strategies, i.e., with strategies that do not require precise global information of the network. Indeed, for social networks, this fact was experimentally confirmed a long time ago by the famous experiment of Travers and Milgram [10] and theoretical explanations have been given by Kleinberg [11] and, more recently, by Watts et al. [12]. These explanations are based on the plausible assumption that there is a structure (social, geographical, etc.) that underlies the complex social network and provides information that can be exploited heuristically in a search process. In scale-free communication networks and in some decentralized peer-to-peer communication networks such as Gnutella or Freenet, it has been shown $[13,14]$ that the skewness of the degree distribution and the existence of highly connected hubs allows the design of algorithms that search quite efficiently even when the size of the system is large.

Our approach in the present work is complementary to these efforts. The question we pose is the following: given a search algorithm that uses purely local informationi.e., knowledge of the first neighbors in the network-and a fixed set of resources -i.e., a fixed number of nodes and links - which is the topology that optimizes the search process? We consider a general situation where the net- work has to tackle several simultaneous (or parallel) search problems, which in turn raises the important issue of congestion [15-18] at overburdened nodes. Indeed, for a single search problem the optimal network is clearly a highly polarized starlike structure. This structure is cheap to assemble in terms of number of links and efficient in terms of searchability, since the average cost (number of steps) to find a given node is always bounded (two steps), independently of the size of the system. However, the polarized starlike structure will become inefficient when many search processes coexist in parallel in the network, due to the limitation of the central node.

The discovery of optimal structures will be a useful guide to design, redesign, and drive the evolution of communication networks such as peer-to-peer networks, distributed databases, and organizations.

In this Letter we present a formalism that is able to cope with search and congestion simultaneously, allowing the determination of optimal topologies. This formalism avoids the problem of simulating the dynamics of the search-communication process which turns out to be impracticable, specially close to the congestion point where search costs (time) diverge. We do not focus on detailed models of any of the above mentioned communication networks (organizations, computer networks, etc.). Rather, we study a general scenario applicable to any communication process. First, we calculate the average number of steps (search cost) needed to find a certain node in the network given the search algorithm and the topology of the network. The calculation is exact if the search algorithm is Markovian. Next, congestion is introduced assuming that the network is formed by nodes that behave like queues, meaning that they are able to deliver a finite number of packets at each time step $[16,17,19]$. In this context, we are able (i) to calculate explicitly the point at which the arrival rate of packets 
leads to network collapse, in the sense that the average time needed to perform a search becomes unbounded, and (ii) to determine, below the point of collapse, how the average search time depends on the rate at which search processes are started. In both cases, the relevant quantities are expressed in terms of the topology of the network and the search algorithm. Finally, we obtain optimal structures by performing exhaustive generalized simulated annealing $[20,21]$ in the space of the networks with fixed size and connectivity. We find that when the number of parallel searches is small, the starlike configuration turns out to be optimal as expected, while for a large number of parallel searches, a very decentralized and uniform network is best. Surprisingly, no other structures apart from these extremely centralized and extremely decentralized networks are found to be optimal.

First, we consider the average cost to find a given node in an arbitrary communication network when there is no congestion. Specifically, we focus on a single information packet at node $i$ whose destination is node $k$, i.e., a packet searching for $k$. The probability for the packet to go from $i$ to a new node $j$ in its next movement is $p_{i j}^{k}$. In particular, $p_{k j}^{k}=0 \forall j$ so that the packet is removed as soon as it arrives at its destination. The precise form of $p_{i j}^{k}$ will depend on the search algorithm. In particular, when the search is Markovian, $p_{i j}^{k}$ does not depend on previous positions of the packet. In this case, the probability of going from $i$ to $j$ in $n$ steps is given by

$$
P_{i j}^{k}(n)=\sum_{l_{1}, l_{2}, \ldots, l_{n-1}} p_{i l_{1}}^{k} p_{l_{1} l_{2}}^{k} \cdots p_{l_{n-1} j}^{k} .
$$

Thus defining the matrices $\mathbf{p}^{k}$ and $\mathbf{P}^{k}(n)$ we have

$$
\mathbf{P}^{k}(n)=\left(\mathbf{p}^{k}\right)^{n} .
$$

We next define the effective distance matrices

$$
\mathbf{d}^{k}=\sum_{n=0}^{\infty} n \mathbf{P}^{k}(n)=\sum_{n=0}^{\infty} n\left(\mathbf{p}^{k}\right)^{n}=\left[\left(\mathbf{I}-\mathbf{p}^{k}\right)^{-1}\right]^{2} \mathbf{p}^{k},
$$

whose elements $d_{i j}^{k}$ are the average number of steps needed to go from $i$ to $j$ for a packet traveling towards $k$ [22]. In particular, the element $d_{i k}^{k}$ is the average number of steps needed to find $k$ starting from $i$. When the search algorithm is such that the packets follow minimum paths between nodes, the effective distance will coincide with the topological minimum distance; otherwise, the effective distance between nodes will be, in general, larger than the topological minimum distance. Finally, the average search cost in the network when there is not congestion is

$$
\bar{d}=\frac{\sum_{i, k} d_{i k}^{k}}{S(S-1)},
$$

where $S$ is the number of nodes in the network.

Consider next which is the centrality of each of the nodes in the communication network. First, we calculate the average number of times, $b_{i j}^{k}$, that a packet generated at $i$ and with destination $k$ passes through $j$. According to the previous definitions

$$
\mathbf{b}^{k}=\sum_{n=1}^{\infty} \mathbf{P}^{k}(n)=\sum_{n=1}^{\infty}\left(\mathbf{p}^{k}\right)^{n}=\left(\mathbf{I}-\mathbf{p}^{k}\right)^{-1} \mathbf{p}^{k} .
$$

The effective betweenness of node $j, B_{j}$, is defined as

$$
B_{j}=\sum_{i, k} b_{i j}^{k} \text {. }
$$

Again, as in the case of the effective distance, when the search algorithm is able to find the minimum paths between nodes, the effective betweenness will coincide with the topological betweenness, $\beta_{j}$, as usually defined $[23,24]$. The effective betweenness of the nodes in a network contains valuable information about its behavior when multiple searches are performed simultaneously and congestion considerations become relevant.

Consider the following general scenario. In the communication network, each node generates packets at a rate $\rho$ per unit of time, independently of the rest of the nodes. The destination of each of these packets is randomly fixed at the moment of its creation. On the other hand, the nodes are queues that can store as many packets as needed but can deliver, on average, only a finite number of them at each time step-without loss of generality, we fix this number to 1 . It is known [16-18] that for low values of $\rho$ the system reaches a steady state in which the total number of floating packets in the network $N(t)$ fluctuates around a finite value. As $\rho$ increases, the system undergoes a continuous phase transition to a congested phase in which $N(t) \propto t$ [16]. Right at the critical point, $\rho_{c}$, quantities such as $N(t)$ and the characteristic time diverge [25]. Below $\rho_{c}$, there is no accumulation at any node in the network and the number of packets that arrive at node $j$ is, on average, $\rho B_{j} /(S-1)$. Therefore, a particular node will collapse when $\rho B_{j} /(S-1)>1$ and the critical congestion point of the network will be

$$
\rho_{c}=\frac{S-1}{B^{*}},
$$

where $B^{*}$ is the maximum effective betweenness in the network, which corresponds to the most central node.

To calculate the average of the load of the network, $\langle N(t)\rangle$, it is necessary to establish the behavior of the queues. In the general scenario proposed above, the arrival of packets at a given node $j$ is a Poisson process with mean $\mu_{j}=\rho B_{j} /(S-1)$. Regarding the delivery of packets, assume the simplest case in which it is also a Poisson process and hence the time between two consecutive packet deliveries follows an exponential distribution [26]. In general, when the arrival and delivery processes are Poisson, the average size of the queues is given by $[19,27]$

$$
\left\langle\nu_{j}\right\rangle=\frac{\mu_{j}}{1-\mu_{j}}=\frac{\frac{\rho B_{j}}{S-1}}{1-\frac{\rho B_{j}}{S-1}} .
$$


The average load of the network $\langle N(t)\rangle$ is

$$
\langle N(t)\rangle=\sum_{j=1}^{S}\left\langle\nu_{j}\right\rangle=\sum_{j=1}^{S} \frac{\frac{\rho B_{j}}{S-1}}{1-\frac{\rho B_{j}}{S-1}} .
$$

There are two interesting limiting cases of this expression. When $\rho$ is very small, $\left\langle\nu_{j}\right\rangle \approx \mu_{j}$ and taking into account that $\sum_{j} B_{j}=\sum_{i, k} d_{i k}^{k}$, one obtains

$$
\langle N(t)\rangle \approx \rho S \bar{d} \quad \rho \rightarrow 0 .
$$

On the other hand, when $\rho$ approaches $\rho_{c}$ most of the load of the network comes from the most congested node, and therefore [28]

$$
\langle N(t)\rangle \approx \frac{1}{1-\frac{\rho B^{*}}{S-1}} \quad \rho \rightarrow \rho_{c} .
$$

It is worth noting that there are only two assumptions in the calculations above. The first one has already been mentioned: the movement of the packets needs to be Markovian to define the jump probability matrices $\mathbf{p}^{k}$. Although this is not strictly true in real communication networks - where packets are not allowed usually to go through a given node more than once - it can be seen as a first approximation [16-18]. The second assumption is that the jump probabilities $p_{i j}^{k}$ do not depend on the congestion state of the network, although communication protocols sometimes try to avoid congested regions, and then $B_{j}=B_{j}(\rho)$ [29]. Our calculations, in particular, Eqs. (7)-(11), correspond to the worst case scenario and thus provide bounds to more realistic scenarios in which the search algorithm interactively avoids congestion.

Equations (5), (6), and (9) enable us to tackle the problem of finding optimal structures for local search. Optimality is defined as minimization of the average time needed to perform a search. Indeed, according to Little's law [19], the average time needed by a packet to reach its destination is proportional to the total load of the network, and therefore minimizing $\langle N(t)\rangle$ is equivalent to minimizing the average cost of a search. In a local search scenario, the $\mathbf{p}^{k}$ matrices are given by

$$
p_{i j}^{k}=a_{i k} \delta_{j k}+\left(1-a_{i k}-\delta_{i k}\right) \frac{a_{i j}}{\sum_{l} a_{i l}},
$$

where $a_{i j}$ are the elements of the adjacency matrix of the network. The first term corresponds to $i$ and $k$ being neighbors: then the packet will go to $j$ if and only if $j=k$; i.e., the packet will be sent directly to the destination. The second term corresponds to $i$ and $k$ not being neighbors: in this case, $j$ is chosen at random and uniformly among the neighbors of $i$. Finally, the delta symbol ensures that $p_{k j}^{k}=0 \forall j$ and the packet disappears from the network.

The optimization process is carried out using generalized simulated annealing as described in [20,21]. Starting from a given initial network configuration, random rewiring of individual links is performed, the cost $\langle N(t)\rangle$ is evaluated according to (9), and the change is accepted with a certain probability that depends on a computational temperature, which is decreased so that the system tends to explore regions of the configuration space with lower and lower costs. Regarding the cooling, at a given temperature, each node of the network is allowed to try a rewiring. Then the temperature is decreased by $1 \%$, and the process is repeated until a minimum temperature is reached or, alternatively, the system has remained unchanged after a significantly large amount of rewiring trials. Different sets of initial conditions are explored: for a given value of $\rho$, the optimization process is started from random initial configurations and also from networks that turned out to be optimal at similar values of $\rho$. Of all the realizations, only the network with the smallest cost is considered as optimal.

The results of the optimization process are shown in Fig. 1. For $\rho \rightarrow 0$, the optimal network has a starlike centralized structure as expected, which corresponds to the minimization of the average effective distance between nodes [Eq. (10)]. On the other extreme, for high values of $\rho$, the optimal structure has to minimize the maximum betweenness of the network, according to Eq. (11). This is accomplished by creating a homogeneous network where all the nodes have essentially the same degree, betweenness, etc. To characterize the networks at all values of $\rho$, we introduce a measure of the polarization, $\pi$, of the network:

$$
\pi=\frac{\beta^{*}-\langle\beta\rangle}{\langle\beta\rangle}
$$

where $\beta$ is, as before, the topological betweenness of the nodes. For starlike networks, the value of $\pi$ is large while for very homogeneous networks $\pi \approx 0$. Although one

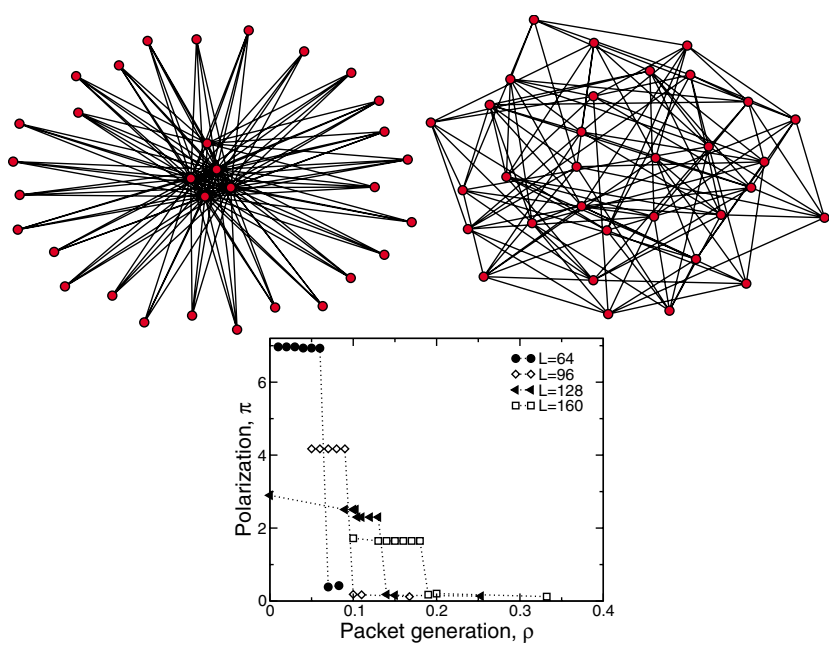

FIG. 1 (color online). Optimal structures for local search with congestion. Top: starlike configuration optimal for $\rho<\rho^{*}$ (left) and homogeneous-isotropic configuration optimal for $\rho>\rho^{*}$ (right). Bottom: polarization of the optimal structure as a function of $\rho$, for networks of size $S=32$ and different number of links $L$. 
could expect that optimal networks cover the whole range of values from $\pi=\pi_{\text {star }}$ to $\pi \approx 0$, the results of the optimization process reveal a completely different scenario. According to simulations, starlike configurations are optimal for $\rho<\rho^{*}$; at this point, the homogeneous networks that minimize $B^{*}$ become optimal. Therefore there are only two type of structures that can be optimal for a local search process: starlike networks for $\rho<\rho^{*}$ and homogeneous networks for $\rho>\rho^{*}$.

In summary, we have found analytical expressions for the relationship between topological properties of networks and the specific dynamic behavior when faced with local search with congestion. These expressions allow the calculation of the search cost in terms of the effective betweenness of the nodes, which is calculated via the transition probability matrices (formal expressions of the search algorithm). This formalism allows one to perform an exhaustive search for optimal topologies in terms of parallel searchability avoiding the simulation of the dynamics of the parallel search process, which is prohibitive in computational time. Moreover, the formalism is general enough to deal with other search scenarios - local searches with knowledge up to second nearest neighbors, third nearest neighbors, and so on (eventually, global knowledge) - simply redefining the $p_{i j}^{k}$ elements. We find that the optimal network topologies for local search considering congestion are split in two categories: a starlike network topology, which is optimal for a small number of parallel searches, and the homogeneous-isotropic network topology, which is optimal for large numbers of parallel searches. Strikingly, the transition between these categories is sharp; i.e., we are not able to find any optimal network topology different from these two classes.

The authors are grateful to L. A. N. Amaral, L. Danon, X. Guardiola, R. Monasson, C. J. Pérez, and M. Sales for helpful comments and discussions. This work has been supported by DGES of the Spanish Government, Grants No. PPQ2001-1519, No. BFM2000-0626, No. BEC20001029, and No. BEC2001-0980, and EC-Fet Open Project No. IST-2001-33555. R. G. also acknowledges financial support from the Generalitat de Catalunya.

[1] D. Watts and S. Strogatz, Nature (London) 393, 440 (1998).

[2] A.-L. Barabasi and R. Albert, Science 286, 509 (1999).

[3] L. A. N. Amaral, A. Scala, M. Barthelemy, and H. E. Stanley, Proc. Natl. Acad. Sci. U.S.A. 97, 11149 (2000).

[4] M. Faloutsos, P. Faloutsos, and C. Faloutsos, Comput. Commun. Rev. 29, 251 (1999).

[5] R. Albert, H. Jeong, and A.-L. Barabasi, Nature (London) 401, 130 (1999).

[6] B. Huberman and L. Adamic, Nature (London) 401, 131 (1999).

[7] R. Radner, Econometrica 61, 1109 (1993).
[8] P. Bolton and M. Dewatripont, Q. J. Econ. 109, 809 (1994).

[9] L. Garicano, J. Political Econ. 108, 874 (2000).

[10] J. Travers and S. Milgram, Sociometry 32, 425 (1969).

[11] J. Kleinberg, Nature (London) 406, 845 (2000).

[12] D. J. Watts, P. S. Dodds, and M. E. J. Newman, Science 296, 1302 (2002).

[13] L. A. Adamic, R. M. Lukose, A. R. Puniyani, and B. A. Huberman, Phys. Rev. E 64, 046135 (2001).

[14] B. Tadic, Eur. Phys. J. B 23, 221 (2001).

[15] V. Jacobson, in Proceedings of Communications, Architectures \& Protocols: SIGCOMM '88, Stanford, CA, 1988 (Association for Computing Machinery, New York, 1988).

[16] A. Arenas, A. Diaz-Guilera, and R. Guimera, Phys. Rev. Lett. 86, 3196 (2001).

[17] T. Ohira and R. Sawatari, Phys. Rev. E 58, 193 (1998).

[18] R. Sole and S. Valverde, Physica (Amsterdam) 289A, 595 (2001).

[19] O. Allen, Probability, Statistics and Queueing Theory with Computer Science Application (Academic Press, New York, 1990), 2nd ed.

[20] T. J. P. Penna, Phys. Rev. E 51, R1 (1995).

[21] C. Tsallis and D. A. Stariolo, Annual Review of Computational Physics II, edited by D. Stauffer (World Scientific, Singapore, 1994).

[22] It is assumed that the eigenvalues of the $\mathbf{p}^{k}$ matrix are smaller than 1, which must be true if the number of times that a packet goes through a certain node is finite.

[23] L. C. Freeman, Sociometry 40, 35 (1977).

[24] M. E. J. Newman, Phys. Rev. E 64, 016132 (2001).

[25] R. Guimera, A. Arenas, A. Diaz-Guilera, and F. Giralt, Phys. Rev. E 66, 026704 (2002).

[26] For example, consider the following model which is a simplification of the communication model with discrete time proposed in Ref. [16]. For a node $j$ with $\nu_{j}$ packets stored in its queue, every time step each packet jumps to the next node (chosen according to the search algorithm defined through the matrices $\mathbf{p}^{k}$ ) with probability $1 / \nu_{j}$. In this simple case, the delivery of packets can be approximated by a Poisson process. The continuum approximation holds in this case because the maximum rate of arrival and delivery is one packet per time step and, therefore, the results obtained in queuing theory can be applied safely. However, the approximation would not hold for discrete models if larger time windows were considered.

[27] Note that, to obtain this result, the order in which packets are processed is irrelevant. Moreover, it is straightforward to extend the calculations to other types of queues.

[28] We assume that there is only one node that has betweenness $B^{*}$. If there is more than one, Eq. (11) should be multiplied by the number of such nodes.

[29] All the derivations above will still be true in a number of general situations, including situations in which the paths that the packets follow are unique [16], in which the routing tables are fixed, or situations in which the structure of the network is very homogeneous and thus the congestion of all the nodes is similar. 